\title{
Neuroanatomical tract-tracing methods beyond 2000: what's now and next
}

Looking back at the great events that took place in the 20th century within the discipline of neuromorphology, it is evident that two periods of great acceleration can be distinguished, one at the very beginning and one at the end of this remarkable century. The first of these periods, the Golgi/Cajal revolution, actually started in the last decade of the 19th century and it moved on at full speed during the first three decades of the next century. The initial discovery by Camillo Golgi and the intelligent exploitation by Santiago Ramon y Cajal and co-workers of what became known as the Golgi silverimpregnation technique, caused an enormous increase in the number of neuromorphological studies. The final outcome of these studies was the wide and final acceptance by the neuroscience community of the neuron doctrine. The second or tracing revolution saw its birth during the late 1960s and accelerated to full momentum in the early 1970s. Like its Golgi/Cajal predecessor, this revolution was set in motion by technological innovation, notably the use of naturally occurring cellular transport for fiber tracing purposes. This second revolution has provided the solid neuroanatomical background for many concepts of brain function. Both periods of acceleration in the 20th century can thus be characterized as being 'technology-driven', and both resulted in a spectacular increase in understanding of the anatomy of the brain.

When we concentrate on the second period of rapid progress, we note that an amazing array of tracing methods and tracers has been introduced, together with other innovations like immunocytochemistry and electron microscopy. Researchers are now facing a quickly changing scenario in which the boundaries between classical disciplines such as neuroanatomy, neurochemistry, neurophysiology and neuropharmacology can no longer be maintained. Information becoming available from marriages between these fields of research is very impressive and therefore the available technical tools have to be adapted to fulfil the requirements inherent to the complex investigations involved.

Although it may be too early, the end of the Decade of the Brain may be seen as a pivotal point. We are finding ourselves in a promising position from which we can look back at what has happened and enjoy the availability of a number of new and impressive techniques. Alternatively, we can look forward and conceive challenging research to further explore the anatomy of the brain and the complex circuitries along which brain areas function.

In the past, comprehensive reviews of new neuroanatomical tracing methods have been compiled. We appreciate the enormous efforts and scientific merit of the neuroscientists that have contributed to the classical textbooks, notably those edited by Cowan and Cuénod (1975), Heimer and Robards (1981), Heimer and Záborszky (1989), Björklund et al. (1990), and Bolam (1992). The detailed information and the careful explanation of every single method described in these books will possibly never fully be acknowledged. Nevertheless, the fast turnover rate with which the arsenal of existing tracers is supplemented with new instruments, makes many protocols quickly obsolete. While retrograde tracers such as horseradish peroxidase (HRP) still rank among the most commonly used neuroanatomical tracers, other powerful retrograde tracers such as Cholera toxin, B subunit or Fluoro-Gold have succeeded this veteran substance. More recently, the versatile anterograde tracer Phaseolus vulgaris leucoagglutinin (PHA-L) has been replaced by biotinylated dextran amine (BDA). The large family of dextran amines probably best symbolizes the success of modern neuroanatomical tracing. Other modern tract-tracing protocols comprise the use of neurotropic viruses for the study of chains of neurons, transported neurotoxins for selective neurolesioning, neuroanatomical tracing in human fixed brain with carbocyanines, and procedures 
aimed at combining tracing protocols and neurophysiological paradigms.

Despite the large amount of potential tracers available, the first, critical question an investigator faces when trying some experimental design involving neuroanatomical tracers, is the tracer selection, i.e. which tracer is or which tracers are best suited to the specific application. The above mentioned anterograde and/or retrograde tracing techniques still do not meet all the requirements for an ideal neuronal tracer and many other tracers or combinations thereof have already been proposed.

In this Special Issue of the Journal of Neuroscience Methods, we deal only with several of the most widely used methods, simply because it is impossible to deal with all available methods. We have tried to put together in one single issue the state-of-the-art protocols most commonly used by the scientific community at large. Our commitment was to provide potential readers with a dynamic issue including the most relevant contributions currently available for the study of brain circuits. Contributions have been made by leading experts in each particular technique or in combinations thereof. Authors have been encouraged to provide detailed recipes of their technical protocols and supplement these with trouble-shooting sections at the end of their contribution in order to facilitate replication of their methods. Both editors would like to express their gratitude to all scientists contributing to the Special Issue and to their respective grant funding agencies. The assistance received from the former Editor-in-Chief of the Journal of Neuroscience Methods (Dr N.G. Bowery), as well as from the newly appointed Editors-inChief (Professor V. Crunelli and Dr G. Gerhardt) and Elsevier's headquarters is also acknowledged.

\section{Acknowledgements}

Supported by PM98-0035, Departamento de Salud del Gobierno de Navarra, Fundación Marcelino Botín and Universidad de Navarra.

\section{References}

Björklund A, Hökfelt T, Wouterlood FG, van den Pol AN, editors. Handbook of Chemical Neuroanatomy, vol. 8. Amsterdam: Elsevier, 1990.

Bolam JP, editor. Experimental Neuroanatomy. Oxford: Oxford University Press, 1992.

Cowan WM, Cuénod M. The Use of Axonal Transport for Studies of Neuronal Connectivity. Amsterdam: Elsevier, 1975.

Heimer J, Robards MJ, editors. Neuroanatomical Tract-Tracing Methods. New York: Plenum, 1981.

Heimer J, Záborszky L, editors. Neuroanatomical Tract-Tracing Methods. Recent Progress. New York: Plenum, 1989.

Departamento de Anatomía, Facultad de Medicina, Universidad de Navarra, Irunlarrea $s / n, 31080$ Pamplona, Spain

F.G. Wouterlood Graduate School Neurosciences Amsterdam, Research Institute Neurosciences Vrije Universiteit, Department of Anatomy and Embryology, Faculty of Medicine, Vrije Universiteit, 7 v.d. Boechorststraat, 1081 BT Amsterdam, The Netherlands E-mail: jlanciego@unav.es 